\title{
Evolutionary gene expression of lactate dehydrogenase in water snakes Cerberus rhynchops and Xenochrophis piscator
}

\author{
P. Senthilkumaar and V. Kalyanaraman \\ School of Enzymology and Environmental Toxicology (SEET) \\ PG \& Research Dept. of Zoology, Sir Theagaraya College, Chennai, Tamilnadu, India. \\ drsenthilkumaar@yahoo.co.in; kalyanvraman@yahoo.co.in
}

\begin{abstract}
Species differences are known to exist with reference to LDH profile. A four-band isoenzyme pattern is a feature in most snakes, which is a deviation from the primitive ancestor. The LDH morphic state of two water snakes, Cerberus rhynchops and Xenochrophis piscator belonging to the same family Colubridae was selected for the geneological understanding since one of the species is a known salt tolerant. The study was undertaken to observe the fractions of the key isoenzyme LDH subunit diversity in an evolutionary backdrop.
\end{abstract}

Keywords: Lactate dehydrogenase, Cerberus rhynchops, Xenochrophis piscator.

Introduction

Lactate dehydrogenase (LDH) (EC 1.1.1.27) is an enzyme present in one form or other in wide variety of organisms including plants and animals. Lactate dehydrogenases are $N A D(P)$ - dependent enzymes with each act on either D-lactate or L-lactate. LDHs are pyridine nucleotide linked and are highly specific either for $D$ or for the $L$ form of lactate. These enzymes catalyse the following overall reactions:

L- (Or D-) lactate $+\mathrm{NAD}^{+} \Leftrightarrow$ pyruvate $+\mathrm{NADH}+\mathrm{H}^{+}$

$\mathrm{LDH}$ is a key enzyme, which plays an important role in several metabolic pathways, and it forms the centre of a delicately balanced homeostasis in the metabolism of carbohydrates. It is a terminal enzyme in the sequence of reactions involved in anaerobic glycolysis promoting the breakdown of glucose to lactate and results in the essential production of ATP.

L-Lactate dehydrogenase is encoded by two or three loci in almost all vertebrates. Biochemical characterizations of LDH proteins have suggested that gene duplication early in vertebrate evolution gave rise to Ldh-A and Ldh-B and that an additional locus, Ldh-C arose in a number of lineages more recently. Although some phylogenetic studies of LDH protein sequences have supported this pattern of gene duplication, others have contradicted it. In particular, a number of studies have suggested that $\mathrm{Ldh}-\mathrm{C}$ represents the earliest divergence among vertebrate LDHs and that it may have diverged from the other loci well before the origin of vertebrates (Stock et al., 1997).

The subunits of lactate dehydrogenase exist as two major structural forms, usually referred to as $\mathrm{M}$ (muscle) and $\mathrm{H}$ (heart) or $\mathrm{A}$ and $\mathrm{B}$, respectively, which give rise to five different isozymes of the tetrameric molecule in higher vertebrates. The differences in the properties of the $\mathrm{LDH}$ isozymes are dependent on their subunit composition and are most exaggerated between the $M_{4}$ or $A_{4}(L D H-5)$ and $H_{4}$ or $B_{4}(L D H-1)$, the other three hetrotetramers that are expressed are $A_{3} B_{1}, A_{2} B_{2}$ and $\mathrm{A}_{1} \mathrm{~B}_{3}$ (Eventoff et al., 1977)

Changes in gene expression are likely to play a critical role in both acclimation and adaptation to a changing environment. There is a rapidly growing body of literature implicating quantitative changes in gene expression during acclimation to environmental change, but less is known about the role of qualitative changes in gene expression, such as switching between alternative isoforms (Schulte, 2004). However, recent data suggest that isoforms arising from alternative splicing may be an under-appreciated source of physiological variation. The role of changes in gene expression during evolutionary adaptation has received comparatively limited attention.

Schwantes (2005) has studied the LDH isoenzyme patterns of thirteen species of snakes by using starch gel electrophoresis. The two loci of LDH interact to form discrete combinations of the four different subunits and these different subunit combinations have been suggested to contain phylogenetic information (Murphy \& Crabtree, 1985). The utility of these data seems to be variable (Murphy, 1988; Sites et al., 1986; Werman, 1997) their usefulness was further evaluated in this study. Materials and methods

The experimental animals Cerberus rhynchops and Xenochrophis piscator, water snakes commonly known as dog faced and checkered keel back respectively were collected from different localities of Poondi canal, Thiruvallur District, Tamilnadu. A total of five individuals, representing the two species in equal numbers were examined.

Adult specimens were brought to the laboratory with utmost care in live condition and the tissues viz. eye lens, brain, trachea, lungs, heart, liver, stomach, intestine, muscle, kidney, vertebrae and skin were dissected. Tissue homogenates were obtained by homogenizing the tissue with phosphate buffer $\mathrm{pH}-7$ and the homogenates were centrifuged at $20000 \mathrm{rpm}$ in $-4^{\circ} \mathrm{C}$. The supernatants
Research article

CIndian Society for Education and Environment (iSee)
"LDH profile to probe evolution in water snakes"

http://www.indjst.org
Senthilkumaar \& Kalyanaraman Indian J.Sci.Technol. 
in micro litres $(\mu \mathrm{L})$ were subjected to study for their $\mathrm{LDH}$ profile adopting polyacrylamide gel electrophoresis (PAGE). The gels were stained for LDH isoenzymes. The experiments were repeated for at least five times and the results obtained were similar. The method employed in the present study is essentially that of Dietz et al. (1972). The relative mobility (Rf) of each subunit of LDH was calculated by applying the criteria of Norment et al. (1972).

\section{Results and discussion}

The results obtained from Cerberus rhynchops showed three LDH compositions; Ldh- $A_{3} B_{1}$, Ldh- $A_{2} B_{2}$ and Ldh- $A_{1} B_{3}$ in eye lens, brain and trachea and all the other tissues showed four LDH compositions; Ldh- $A_{4}$, Ldh$A_{3} B_{1}$, Ldh- $A_{2} B_{2}$ and Ldh- $A_{1} B_{3}$ whereas in Xenochrophis piscator, eye lens, lungs, liver, muscle, kidney, vertebrae and skin revealed four LDH compositions; Ldh- $A_{4}$, Ldh$A_{3} B_{1}$, Ldh- $A_{2} B_{2}$ and Ldh- $A_{1} B_{3}$ and the tissues brain, trachea, heart, stomach and intestine revealed only one LDH composition; Ldh-B 4 (Fig, 1 \& 2). The Rf values for the $\mathrm{LDH}$ composition in $C$. rhynchops were $0.18,0.24$, 0.28 and 0.32 for LDH-5, LDH-4, LDH-3 and LDH-2 respectively and the $\mathrm{Rf}$ values for $X$. piscator were 0.18 , $0.24,0.28,0.32$ and 0.34 for LDH-5, LDH-4, LDH-3, LDH2 and LDH-1 respectively.

A four-banded isoenzyme pattern, not a three-banded one, is regularly observed among amphibians and reptiles (Murphy, 1988). A three-banded isoenzyme pattern, resulting from the asymmetrical heterotetramers, has been reported from Lacertid lizards, some Xantusiid lizards (Sites et al., 1986) and in rattlesnakes (Murphy \& Crabtree, 1985). A four-banded isoenzyme is frequently reported in reptiles, including in the present investigation. In the current study on $C$. rhynchops and $X$. piscator, the five LDH enzyme isoforms have been recorded, but when it comes to a particular species, a four-band pattern is exhibited. It suggests that reptiles evolve from the fivebanded pattern, through the four-banded pattern, to the stage where progressively fewer bands are expressed. The plesiomorphic four-banded state is shared by colubrid snakes Clelia scytalina, Laticauda colubrina, L. laticaudata and Emydocephalus anulatus (Murphy, 1988).

Liu-wang et al. (1995) reported LDH isozymes in four kinds of tissues, in five species of Colubridae: Opheodrys major, Natrix tigrina lateralis, Zaocys dhumnades, Natrix annularis and Dinodon refozonatum. The results indicated that LDH isozyme of snake had tissue specificity and different LDH zymograms in each tissue of the animals. The study was also of the opinion that LDH zymograms may be used to identify species as one kind of index of biochemistry.

$\mathrm{LDH}$ isozyme patterns of different tissues in the following species of snakes, Elaphe dione, E. rufodorsata, E. schrenckii, Amphiesma vibakari, Rhabdophis tigrina, Agkistrodon saxatilis and $A$. ussuriensis, indicated that LDH isozymes exhibit tissue specificity and difference in the same kind tissues in
Vol. 3 No. 4 (Apr. 2010)

ISSN: 0974- 6846

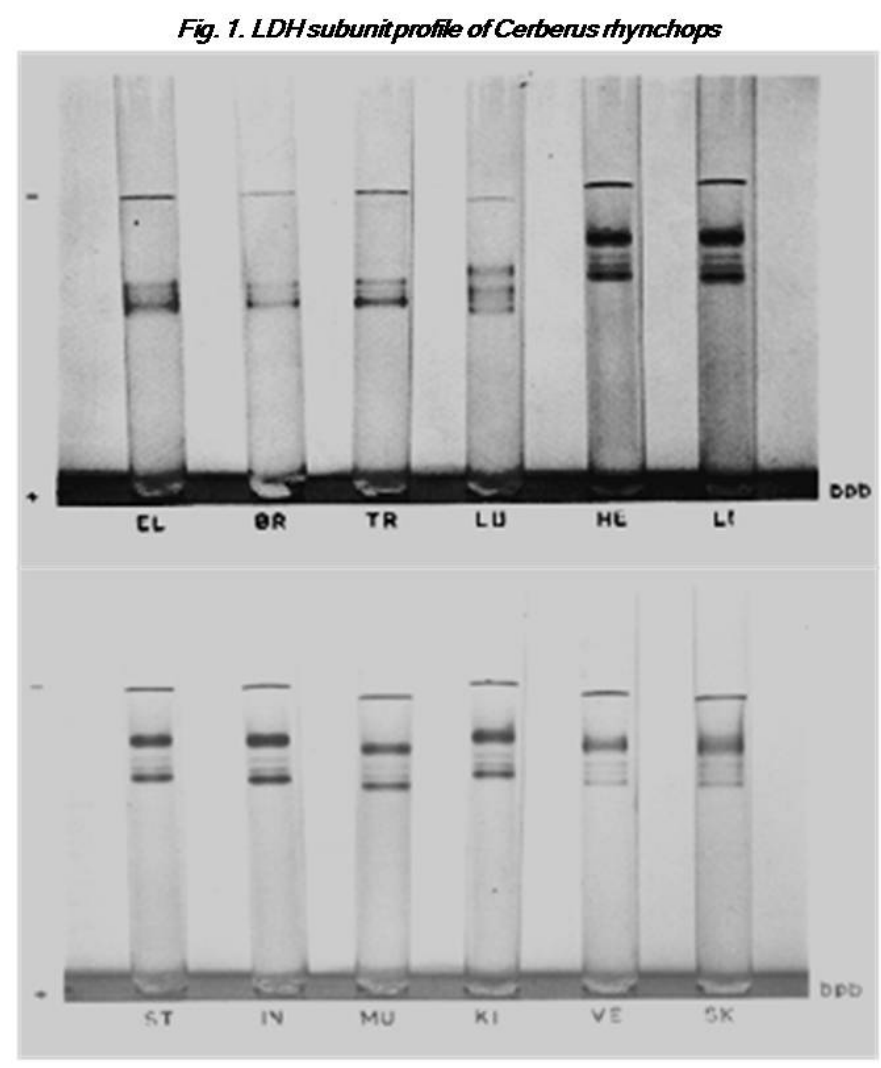

Fig. 2. LDHsubunitprofile of Xenochrophis piscator

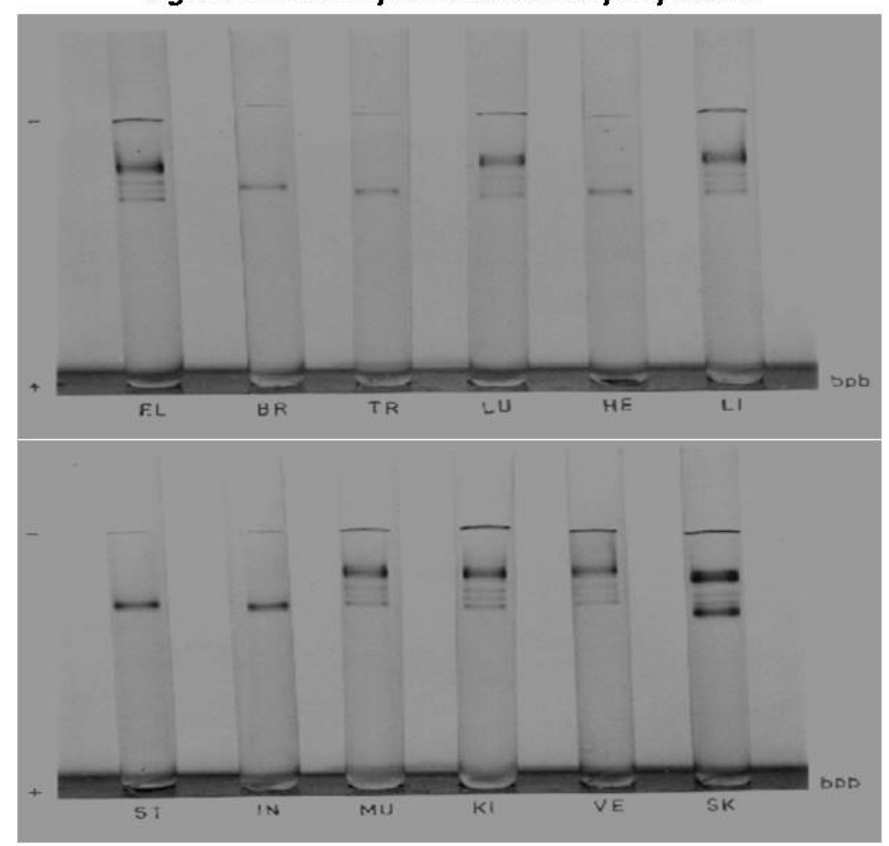

EL-Eye kens; BR-Brain; TR-Trachea; LU-Aungs; HE-Heart LL-Liver, ST-Stomach; IN-ntestine; MU-Muscle; KI-Kidney, E-Vertebrae; SK-Skin

different species. These differences may be used as a biochemical method to distinguish different species of snakes ( $\mathrm{Li}, 1998)$. The LDH isozymes evaluated in the present two species also exhibited tissue specificity, 
some expressing only one fraction and species specificity clearly marked.

Interestingly polymorphs with reference to LDH subunits in snakes are widely reported. In Pseustes sulphureus sulphureus and Clelia clelia plumbea three bands were detected at the LDH-5 site, suggesting the existence of an electro-phoretic variant of the A subunit. Additional isozymes restricted to regions of the eye were also observed in some Bothrops insularis and $B$. jararaca (Schwantes, 2005). Polymorphism for LDH A was registered in a few species of West Indian xenodontine snakes. Thaddeus and Owens (1991) studied the genic similarity in the gray and brown color morphs of the snake Storeria occipitomaculata and found $\mathrm{LDH}-2^{\mathrm{b}}$ allele only in the brown morph. The current investigation on two water snakes failed to detect LDH polymorphim.

Seasonal changes of lactate dehydrogenase isozymes are another feature of this metabolic enzyme. It is noted in Nyctus noctula during hibernation and in Rana nigromaculata during dormancy (Li da-jun, 1994). The catalytic property of LDH in poikilotherms is found to function with the same efficiency at all temperatures within the range of habitat and to adjust immediately to thermal changes (Nelia et al., 1973). Species specific pattern and tissue specificity in the present study and report of polymorphic state in some species and seasonal changes of lactate dehydrogenase isoenzyme suggests that the gene for this pivotal enzyme, involved in energy metabolism expresses according to the metabolic need. These temperature-adaptation processes are evolutionary events in which the evolution and structure of the genetic code are involved.

\section{Conclusion}

Lactate dehydrogenase is known to have multitude of temperature related amino acid substitutions, which is well coded in the genes. The LDH differences in heterotetramers as well as homotetramers in the two species observed augment that the expression of genes Ldh-A and Ldh-B is not the alike in aquatic snakes belonging to the same family (Colubridae). The uniqueness may be attributed to the ability of the individual species to respond to the environmental stress like salt concentration and turbidity. Tissue specificity observed suggests that the LDH gene behavior varies depending upon the energy requirement of the tissue; might be due to the variation in water flow. This flexibility of gene expression makes the animal especially poikilotherms more adaptable to the changing environment. The study is also an ample proof to use the LDH profile as a specific biochemical marker for establishing the species identity.

\section{References}

1. Dietz AA, Lubrano $T$ and Rubinstein HM (1972) Electrophoresis. In: Standard methods in clinical chemistry, Academic Press Inc., NY and London. 7, 49.
2. Eventoff W, Michael GR, Taylor SS, Hans-Joachim T, Helmut M, Walter K and Hans-Hermann K (1977) Structural adaptations of lactate dehydrogenase isozymes. Proc. Nati. Acad. Sci. USA. 74(7), 26772681.

3. Li da-jun (1994) Seasonal changes of lactate dehydrogenase isozymes and blood glucose concentration in Nyctus noctula and Rana nigromaculata. Acta. Physiol. Sinica. 03-08.

4. Li XZW (1998) The LDH Isozyme page analysis of four tissues of five species in Colubridae. Nat. Sci. J.. Harbin Normal Univ. 1-14.

5. Lie-Wang N, Guo CW and Wu XB (1995) The LDH isozyme PAGE analyais of four tissues of five species in Colubridae. Zoo. Res. 16(1), 31-36.

6. Murphy RW (1988) The problematic phylogenetic analysis of interlocus heteropolymer isozyme characters: A case study from sea snakes and cobras. Can. J. Zoo. 66, 2628-2633.

7. Murphy RW and Crabtree BC (1985) Evolutionary aspects of isozyme patterns, number of loci, and tissue-specific gene expression in the prairie rattlesnake, Crotalus viridis viridis. Herpetologica. 41,451-470.

8. Nelia MG, Carlos B, Mercedes G and Blanco A (1973) Effect of temperature upon catalytic properties of lactate dehydrogenase isoenzymes from a poikilotherm. Biochimica. et Biophysica. Acta. (BBA)Enzymol. 315(2), 1250-1258.

9. Norment BR, Haskins AR and Hebner LW (1972) A comparative electrophoretic study of Cicadeuidae and Membracidae. Ann. Entomol. Soc. Amer. 65, 11491152.

10.Schulte PM (2004) Changes in gene expression as biochemical adaptations to environmental change. Comparative Biochemistry and Physiology Part B: Biochem. Mol. Biol. 139(3), 519-529.

11.Schwantes ML (2005) Lactate dehydrogenase isoenzyme patterns of thirteen species of snakes. $J$. Exp. Zool. 185, 3411-316.

12.Sites JW, Bezy RL and Thompson P (1986) Nonrandom expression of lactate dehydrogenase isozymes in the lizard family Xantusiidae. Biochem. System. Ecol. 14, 539-545.

13.Stock DW, Quattro, Whitt GS and Powers DA (1997) Lactate dehydrogenase (LDH) gene duplication during chordate evolution: the CDNA sequence of the LDH of the tunicate Styela plicata. Mol. Biol. Evol. 14, 12731284.

14. Thaddeus AG and Owens PJ (1991) Genic similarity in the gray and brown color morphs of the snake Storeria occipitomaculata. J. Herpatol. 25(1), 90-92.

15.Werman SD (1997) Lactate dehydrogenase variation in Neotropical pitvipers. In: Venomous snakes: Ecology, evolution and snakebite. Thorpe RS, Wuster W \& Malhotra A (Eds.), Clarendon Press, Oxford. p; 79-88. 